\title{
Evolution of sweet taste perception in hummingbirds by transformation of the ancestral umami receptor
}

\section{Citation}

Baldwin, M. W., Y. Toda, T. Nakagita, M. J. O'Connell, K. C. Klasing, T. Misaka, S. V. Edwards, and S. D. Liberles. 2014. "Evolution of Sweet Taste Perception in Hummingbirds by Transformation of the Ancestral Umami Receptor." Science 345 (6199) (August 21): 929-933. doi:10.1126/ science. 1255097.

\section{Published Version}

doi:10.1126/science. 1255097

\section{Permanent link}

http://nrs.harvard.edu/urn-3:HUL.InstRepos:14872099

\section{Terms of Use}

This article was downloaded from Harvard University's DASH repository, and is made available under the terms and conditions applicable to Other Posted Material, as set forth at http:// nrs.harvard.edu/urn-3:HUL.InstRepos:dash.current.terms-of-use\#LAA

\section{Share Your Story}

The Harvard community has made this article openly available.

Please share how this access benefits you. Submit a story.

Accessibility 


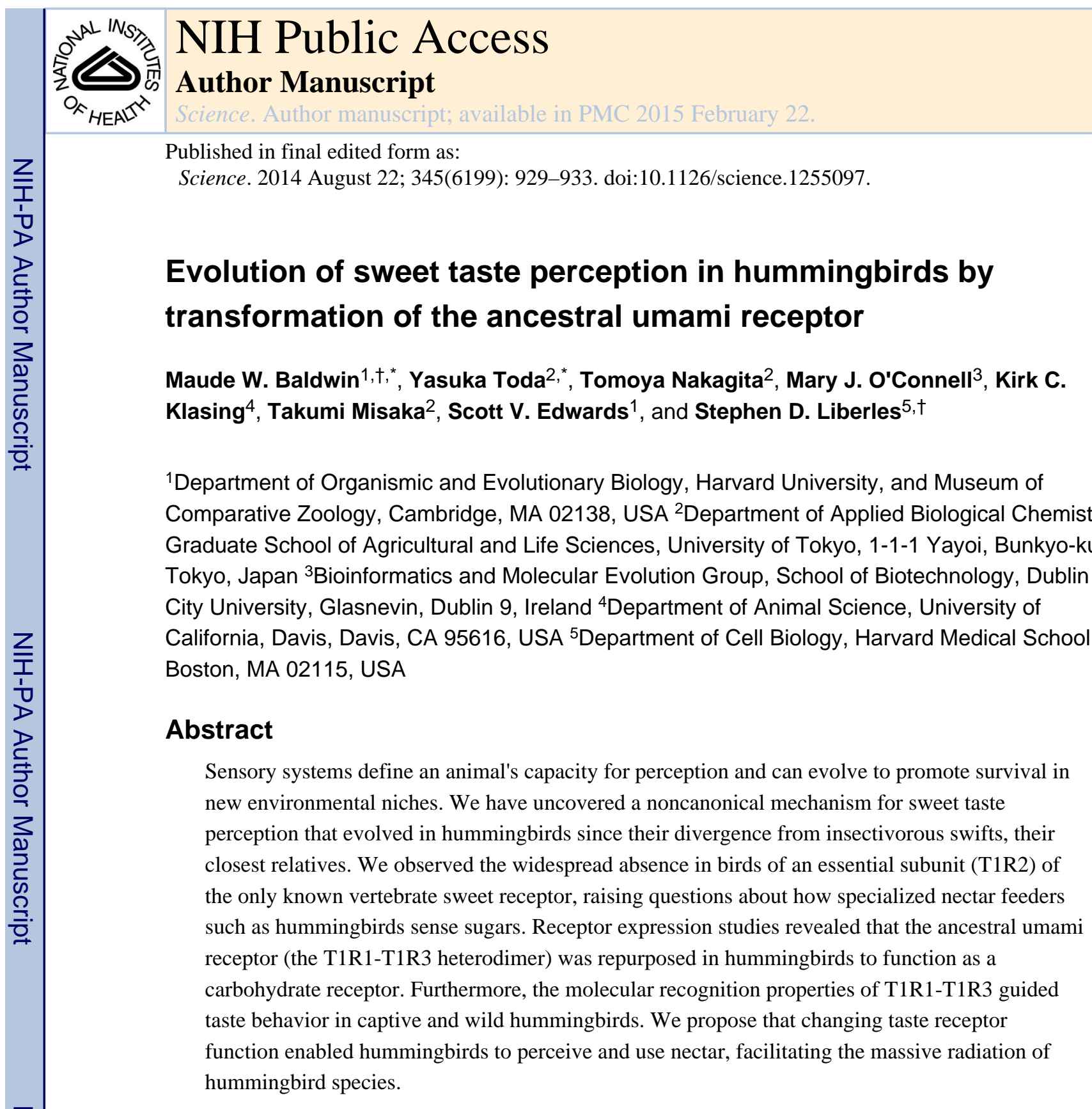

\begin{abstract}
Sensory systems display remarkable flexibility across vertebrates, with some animals losing sensory modalities that are no longer key for survival $(1,2)$ and others evolving new adaptive sensory capabilities (3). The repertoires of sensory receptors for odors, pheromones, and tastes reflect species-specific ecology, with receptor families rapidly
\end{abstract}

Copyright 2014 by the American Association for the Advancement of Science; all rights reserved.

${ }^{\dagger}$ Corresponding author. maudebaldwin@gmail.com (M.W.B.); stephen_liberles@hms.harvard.edu (S.D.L.).

*These authors contributed equally to this work.

SUPPLEMENTARY MATERIALS

www.sciencemag.org/content/345/6199/929/supp1/DC1

Materials and Methods

Supplementary Text

Figs. S1 to S6

Tables S1 to S4

References (26-44)

Movies S1 and S2 
expanding and contracting (4), and in some lineages, new receptor families evolving (5). In the olfactory system, functional expansion of the receptor repertoire predominantly involves a pattern of gene duplication and mutation, leading to novel receptors with altered ligand recognition properties (6). This pattern of gene duplication and mutation is also observed in vomeronasal receptors and bitter taste receptors, but not in sweet and savory taste receptors $(4,7)$. Receptors for these palatable tastes are unique among the chemosensory receptor families in that they are highly conserved in number and amino acid identity. New vertebrate sweet receptors, and the evolutionary mechanisms that underlie their acquisition, have not previously been identified.

In vertebrates, sweet and savory ("umami") tastes are sensed by G protein-coupled receptors (GPCRs) termed T1Rs (8). Most vertebrates have three T1Rs, with the T1R1-T1R3 heterodimer mediating umami taste and the T1R2-T1R3 heterodimer mediating sweet taste $(8,9)$. Human T1R2-T1R3 detects carbohydrates and artificial sweeteners (10), and knockout mice lacking T1R2 or T1R3 have defective sweet taste perception $(9,11)$. In genomes analyzed so far, $T 1 R$ expansions are observed only in some fish species (12), whereas losses are observed in other vertebrates, often in accordance with diet. Some obligate carnivores, such as cats, lost T1R2 and appetitive behaviors toward carbohydrates (1), whereas the giant panda, which feeds predominantly on bamboo, lost T1R1 (2). Chickens, turkeys, and zebra finches also do not have T1R2 (13), but the relationship between T1R repertoire and avian ecology is unclear. Birds display tremendous heterogeneity in diet, with different lineages primarily consuming fruits, nectars, animals, and seeds. Hummingbirds are specialized nectar feeders, and their ability to perceive and use sugar-rich resources allowed them to colonize a nectarivorous niche, enabling their extensive diversification (14). However, how hummingbirds detect sugars remains unknown, so we characterized the repertoires and functions of bird taste receptors to understand the underlying mechanisms of sugar perception.

We identified T1Rs in whole-genome sequences available for 10 birds with different diets and compared them to T1Rs from other vertebrates (Fig. 1A). Also, we cloned T1Rs from the oral tissue of Anna's hummingbirds (Calypte anna); the domestic chicken (Gallus gallus), which does not prefer sugars (7); and the insectivorous chimney swift (Chaetura pelagica), because swifts are the closest living relatives of hummingbirds (Fig. 1B and fig. $\mathrm{S} 2)$. Expression in oral tissue was verified by reverse transcription polymerase chain reaction (fig. S2). Two $T 1 R$ genes-TIR1 and $T 1 R 3$-were detected in each available bird genome, and candidate signatures of positive selection were identified in the hummingbird lineage (Fig. 1B and table S2), but not the chicken or swift lineages. We failed to detect $T 1 R 2$ in bird genomes, despite the presence of flanking loci. Non-avian reptiles retained $T 1 R 2$, including the Chinese alligator (Alligator sinensis), a member of the sister group to birds (fig. S1), suggesting that the loss of $T 1 R 2$ occurred within Dinosauria. These findings suggest that an alternative T1R2-independent mechanism for sugar detection arose in avian species that display high behavioral affinity for nectar or sweet fruit.

To identify avian sweet receptors, we analyzed responses of bird taste receptors to sugars and amino acids (Fig. 2). Responses of bird T1Rs were measured in heterologous cells by means of calcium-sensitive photoprotein reporters (15). Hummingbird T1R1-T1R3 
responded to several carbohydrates, including sucrose, fructose, and glucose. Responses were not observed when T1R1 or T1R3 alone was used, suggesting that hummingbird T1R1-T1R3 functions as an obligate heterodimer. Hummingbird T1R1-T1R3 also detected sucralose and various sugar alcohols, including sorbitol and erythritol, but not cyclamate, acesulfame K, and aspartame, which are sweet to humans (16). Low-affinity responses were observed to some amino acids, as with the human sweet receptor, which recognizes carbohydrates as well as proteins, dipeptides, and amino acids (8). In contrast, cells expressing chicken or swift T1R1-T1R3 failed to detect carbohydrates at any concentration tested and instead recognized alanine and serine. Thus, T1R1-T1R3 heterodimers from swifts, chickens, primates (humans, squirrel monkeys, baboons, and macaques), rodents (mouse and rat), and teleost fish (zebrafish and medaka) detect palatable amino acids (8, 17, 18). In contrast, in the hummingbird lineage, this receptor complex acquired a new function in the past 42 to 72 million years $(14,19)$, evolving the capacity for carbohydrate recognition.

Next, we sought to understand the critical changes in hummingbird T1R1-T1R3 that enabled sugar detection. We designed protein chimeras involving portions of hummingbird T1R1T1R3, which responds to sugars, and chicken T1R1-T1R3, which does not (Fig. 3 and fig. S3). We focused on the venus flytrap domain, an extracellular region of family C GPCRs that mediates ligand binding (20). Introducing the venus flytrap domain of chicken T1R3 into hummingbird T1R3 (chimera 1) rendered the heterodimeric receptor sensitive to amino acids rather than sugars. Reintroducing 109 amino acids (residues 158 to 266) of hummingbird T1R3 into the chicken T1R3 venus flytrap domain restored sucrose responses (chimera 2). Further analysis of this 109-amino acid region identified 19 nonconsecutive amino acids (chimera 3; sites: fig. S3), which were collectively sufficient to impart sucrose and sucralose sensitivity (fig. S4). Subsets of these 19 residues did not similarly support sugar binding (fig. S3). Two identified sites (I206 and S237) displayed evidence of positive selection (Fig. 1B). Hummingbird T1R1 also contains sites that are under putative positive selection and mutations that contribute to acquired sugar responsiveness, because a mixed receptor pair of chicken T1R1 and hummingbird T1R3 prefers amino acids (fig. S5). Thus, the evolution of carbohydrate detection by hummingbird T1R1-T1R3 involved widespread mutation of both receptor subunits.

We created a homology model of the T1R3 venus flytrap domain based on the x-ray crystal structure of the same region in a related GPCR, metabotropic glutamate receptor 1 (mGluR1) (20) (Fig. 3D). This model predicted that the 19 sugar response-conferring substitutions in T1R3 were clustered in three distinct regions of the protein. Three residues (G165, I167, and N211) were in the putative orthosteric ligand-binding site, which in mGluR1 is located at the interface between the two lobes of the venus flytrap domain on the extracellular surface (20). G165 and I167 align near S186 and T188 of mGluR1, which form salt bridges to the glutamate ligand (20), and all three align near T1R1 residues that are important for ligand responses $(18,21)$. The remaining residues clustered in two other locations, whose functions in family C GPCRs are unknown but may be important for folding topology, interdomain or intersubunit interactions, or $\mathrm{G}$ protein activation. The dramatic redecoration of the T1R1-T1R3 protein surface that occurred in hummingbirds to 
allow for sugar binding makes sense, given that carbohydrates and amino acids adopt completely different structures.

We next asked whether T1R1-T1R3 function would dictate hummingbird taste behavior. We reasoned that nonnutritive agonists of T1R1-T1R3 without caloric value would be palatable to hummingbirds, like artificial sweeteners are to humans. Hummingbirds prefer sugars (22), but behavioral responses to many other human sweeteners are unknown. We developed a brief-access, two-choice gustatory preference paradigm in captive ruby-throated hummingbirds (Archilochus colubris) to measure taste responses to T1R1-T1R3 ligands (Fig. 4A). As expected, hummingbirds displayed strong behavioral affinity for sucrose over water, as measured by an increase in mean drinking bout length, number of long bouts ( $>1 \mathrm{~s}$ of uninterrupted drinking), and overall time spent drinking. High-speed video recordings (movie S1) indicated extremely rapid choice decisions; water trials terminated within three or four tongue licks ( $250 \mathrm{~ms}$ ), suggesting that sugar preference involves rapid processing of taste information rather than post-ingestive effects. Ruby-throated hummingbirds equally consumed solutions of sucrose and erythritol, a nonnutritive agonist of hummingbird T1R1T1R3, but displayed a strong preference for sucrose over aspartame, a sweetener to humans that failed to activate hummingbird T1R1-T1R3.

We also developed a behavioral assay involving Anna's hummingbirds because we cloned T1R1 and T1R3 from this species (Fig. 4B and movie S2). Experiments were performed in the Santa Monica Mountains at a field site frequented by wild hummingbirds. We recorded the behavior of birds presented for 15 min with test stimuli, and in control experiments, hummingbirds displayed strong preference for sucrose over water and high behavioral affinity for several sugars abundant in nectar, including sucrose, glucose, and fructose. Next, we presented solutions of sucrose and test stimuli, and measured the mean drinking bout length. Anna's hummingbirds displayed a strong behavioral attraction to the T1R1-T1R3 agonists erythritol and sorbitol, with responses similar to those to sucrose. In contrast, Anna's hummingbirds displayed a strong preference for sucrose over other structurally diverse human sweeteners that failed to activate hummingbird T1R1-T1R3, including aspartame, cyclamate, and acesulfame K. Furthermore, these synthetic human sweeteners were aversive at high concentrations, because birds rejected mixed solutions containing these chemicals and sucrose (fig. S6 and movie S2) and often displayed a characteristic behavioral pattern involving beak withdrawal, head shaking, and/or spitting that was previously observed in response to the ingestion of bitter plant metabolites (23). This reaction was also observed toward sucralose solutions, and mixtures of sucrose and sucralose were not consumed (fig. S6), indicating that sucralose is also actively rejected. Other species of hummingbirds (black-chinned and Allen's hummingbirds) visited and displayed similar taste preferences (fig. S6). Together, these behavioral experiments show that several agonists of hummingbird T1R1-T1R3, including simple sugars and sugar alcohols, evoke fast, appetitive gustatory responses in hummingbirds. Other synthetic human sweeteners that do not activate hummingbird T1R1-T1R3 are not similarly attractive and are often actively rejected. We conclude that the molecular recognition properties of hummingbird T1R1-T1R3, together with those of other gustatory receptors, instruct taste behavior in both captive and wild hummingbirds. We studied the repertoire and function of 
taste receptors to provide a molecular basis for variations in animal ecology and the evolutionary events that cause them. We identified a transformation of taste receptor function that occurred in hummingbirds after their divergence from an insectivorous ancestor. We propose this to be a key evolutionary adaptation that contributed to the acquisition of nectar-feeding behavior and enabled the extensive radiation of hummingbird species. The molecular basis for this change in taste behavior is an altered ligand-binding preference of T1R1-T1R3 from amino acids to carbohydrates, a complex feat that involved dramatic structural changes in the receptor surface. It has been proposed that the ancestral T1R heterodimer, as well as the ancestral family C GPCR, were amino acid receptors (17, 24). The mammalian sweet receptor probably derived from a similar transformation that occurred earlier in vertebrates. Birds descended from carnivorous theropod dinosaurs (25), and like mammalian carnivores, it appears that an ancestor of birds lost T1R2, perhaps another example of the close relationship between diet and taste receptor repertoire. Based on evidence presented here, hummingbirds recently evolved a new sugar receptor and consequently regained sweet taste perception.

\section{Supplementary Material}

Refer to Web version on PubMed Central for supplementary material.

\section{ACKNOWLEDGMENTS}

We thank those who supported these experiments (see the supplementary materials for the full list). Grants to M.W.B. (NSF grant DDIG 1110487, Society for Integrative and Comparative Biology, and Sigma Xi); M.J.O'C. (Fulbright Commission and Science Foundation Ireland Research Frontiers Program, grant EOB2673); S.D.L. (NIH, grant RO1DC013289); S.V.E. (NSF); and T.M. (Japan Society for the Promotion of Science, grant LS037) are acknowledged. T1R sequences and alignments are available at GenBank and Dryad.

\section{REFERENCES AND NOTES}

1. Jiang P, et al. Proc. Natl. Acad. Sci. U.S.A. 2012; 109:4956-4961. [PubMed: 22411809]

2. Zhao H, Yang JR, Xu H, Zhang J. Mol. Biol. Evol. 2010; 27:2669-2673. [PubMed: 20573776]

3. Gracheva EO, et al. Nature. 2010; 464:1006-1011. [PubMed: 20228791]

4. Nei M, Niimura Y, Nozawa M. Nat. Rev. Genet. 2008; 9:951-963. [PubMed: 19002141]

5. Liberles SD, et al. Proc. Natl. Acad. Sci. U.S.A. 2009; 106:9842-9847. [PubMed: 19497865]

6. Ferrero DM, et al. ACS Chem. Biol. 2012; 7:1184-1189. [PubMed: 22545963]

7. Shi P, Zhang J. Mol. Biol. Evol. 2006; 23:292-300. [PubMed: 16207936]

8. Yarmolinsky DA, Zuker CS, Ryba NJ. Cell. 2009; 139:234-244. [PubMed: 19837029]

9. Zhao GQ, et al. Cell. 2003; 115:255-266. [PubMed: 14636554]

10. Nelson G, et al. Cell. 2001; 106:381-390. [PubMed: 11509186]

11. Damak S, et al. Science. 2003; 301:850-853. [PubMed: 12869700]

12. Ishimaru Y, et al. Mech. Dev. 2005; 122:1310-1321. [PubMed: 16274966]

13. Feng P, Zhao H. Chin. Sci. Bull. 2013; 58:2198-2204.

14. McGuire JA, et al. Curr. Biol. 2014; 24:910-916. [PubMed: 24704078]

15. Toda Y, Okada S, Misaka T. J. Agric. Food Chem. 2011; 59:12131-12138. [PubMed: 21981007]

16. Glaser D. Pure Appl. Chem. 2002; 74:1153-1158.

17. Oike H. J. Neurosci. 2007; 27:5584-5592. [PubMed: 17522303]

18. Toda Y. J. Biol. Chem. 2013; 288:36863-36877. [PubMed: 24214976] 
19. Brown, JW.; Mindell, DP. The Timetree of Life. Hedges, S.; Kumar, S., editors. Oxford: Oxford Univ. Press; 2009. p. 454-456.

20. Kunishima N, et al. Nature. 2000; 407:971-977. [PubMed: 11069170]

21. Zhang F, et al. Proc. Natl. Acad. Sci. U.S.A. 2008; 105:20930-20934. [PubMed: 19104071]

22. Medina-Tapia N, Ayala-Berdon J, Morales-Pérez L, Melo LM, Schondube JE. Comp. Biochem. Physiol. A Mol. Integr. Physiol. 2012; 161:307-314. [PubMed: 22155281]

23. Kessler D, Baldwin IT. Plant J. 2007; 49:840-854. [PubMed: 17316174]

24. Kuang D, et al. Proc. Natl. Acad. Sci. U.S.A. 2006; 103:14050-14055. [PubMed: 16966606]

25. Dyke, G.; Kaiser, G. Living Dinosaurs: The Evolutionary History of Modern Birds. Chichester, UK: Wiley-Blackwell; 2011. 


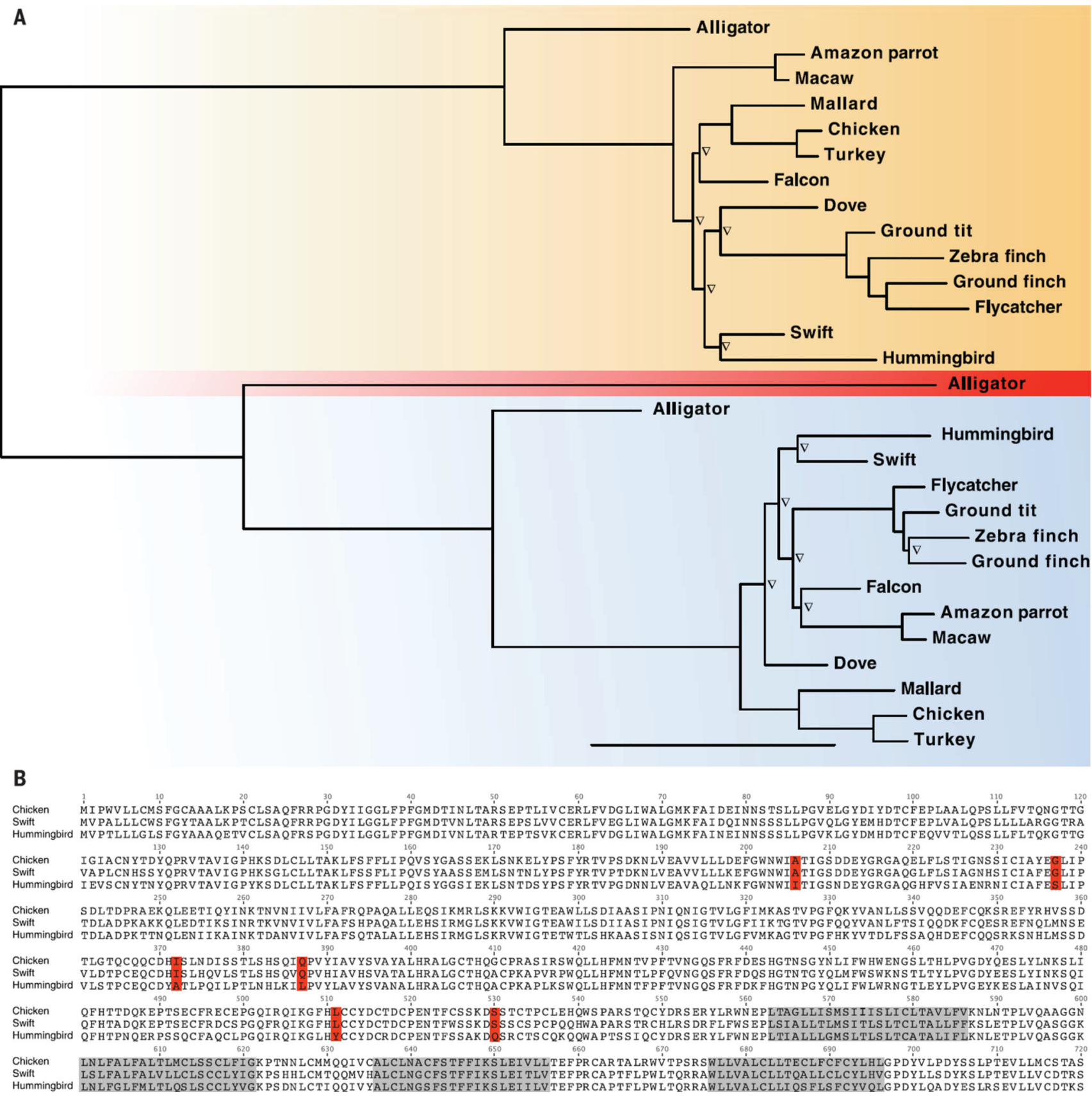

Fig. 1. Analysis of T1R sequences in birds

(A) A maximum-likelihood tree was constructed using $T 1 R$ sequences from 13 birds and the Chinese alligator $(\nabla=$ nodal bootstrap $<80 \%$; scale bar, 0.4 substitutions per site). (B) Amino acid sequences of T1R3 cloned from birds. Gray, transmembrane domains; red, putatively selected sites (table S3). 

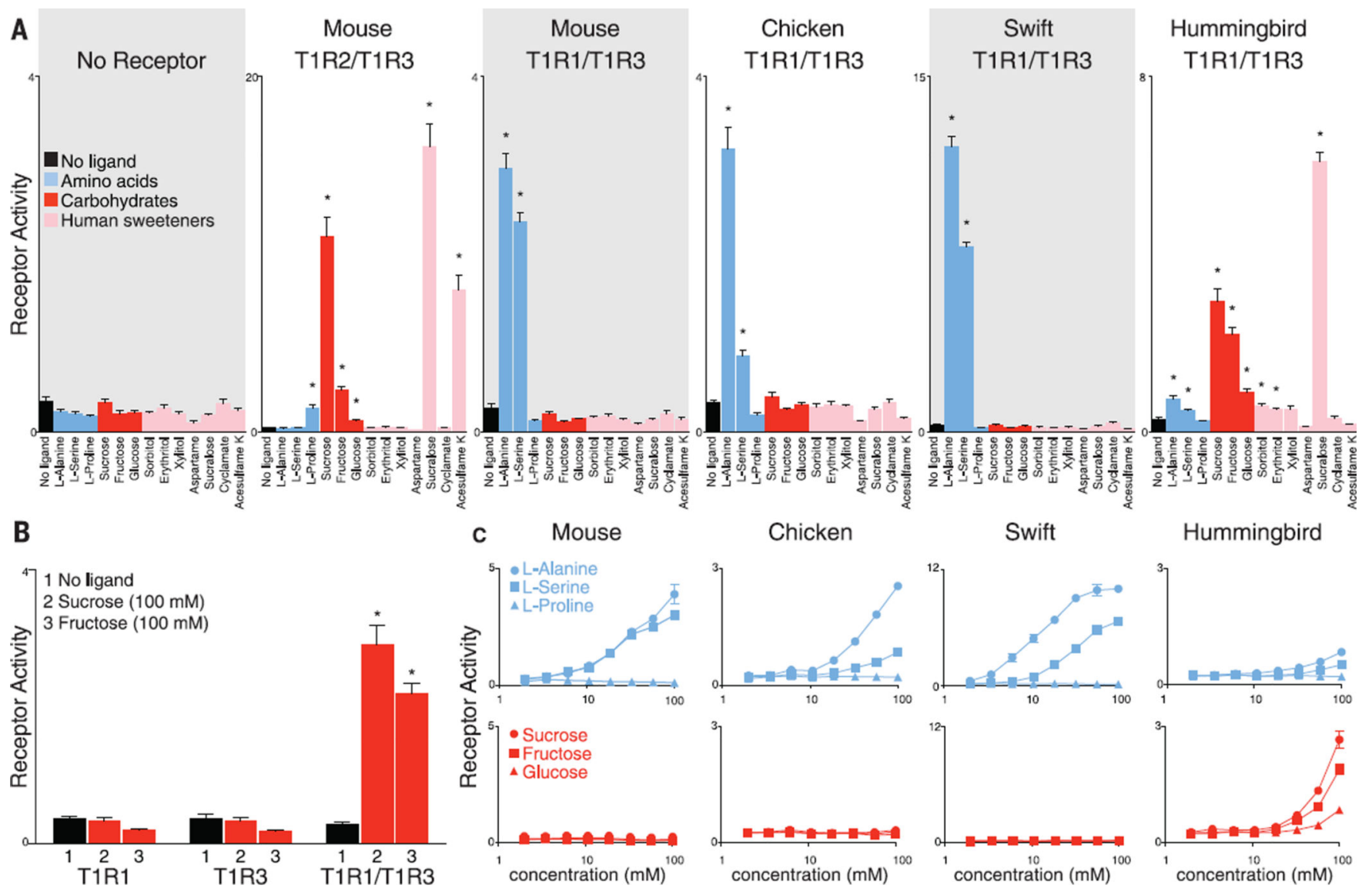

Fig. 2. Evolution of a sugar receptor in hummingbirds

(A) Functional expression of avian and rodent taste receptors to stimuli [100 mM, except aspartame (15 mM); $n=6$ independent experiments, mean $\pm \mathrm{SE}, * P \leq 0.05]$. (B) Sugar responses of hummingbird T1Rs alone or in combination ( $n=6$ independent experiments, mean $\pm \mathrm{SE}, * P \leq 0.05)$. (C) Dose-dependent responses of T1R1-T1R3 from species indicated to amino acids (blue) and sugars (red). 
A

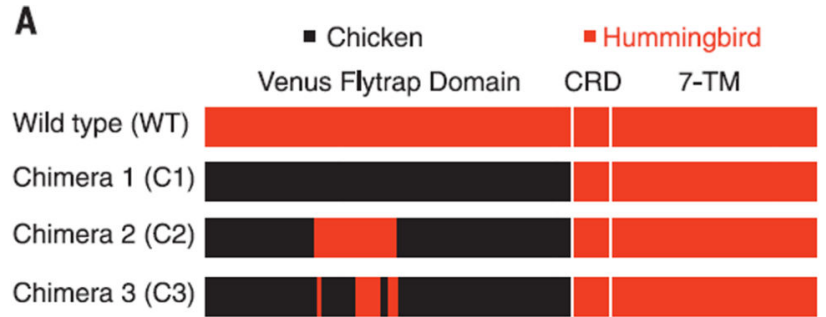

B

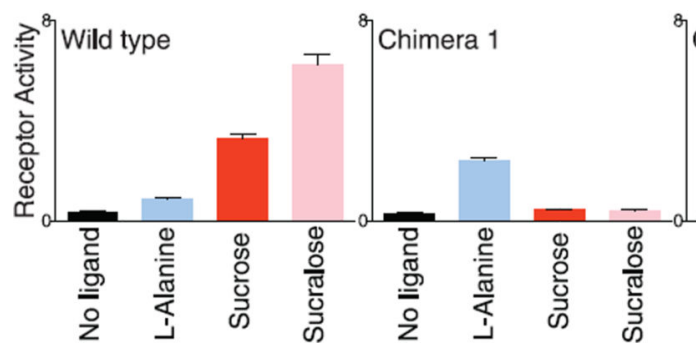

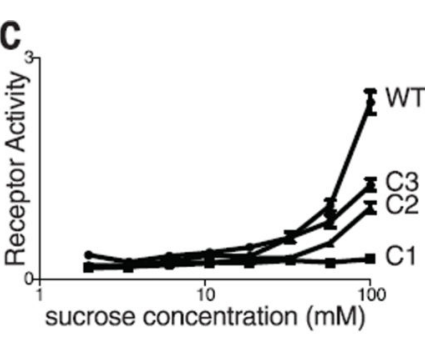

D

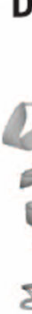

Fig. 3. Molecular basis for the acquisition of sugar binding in hummingbird T1R1-T1R3

(A) T1R3 chimeras containing chicken (black) and hummingbird (red) amino acids were designed (CRD, cysteine-rich domain; TM, transmembrane domains). (B) Responses of T1R3 chimeras and hummingbird T1R1 to L-alanine, sucralose, and sucrose (100 mM). (C) Dose-dependent responses of T1R3 chimeras and hummingbird T1R1 to sucrose. (D) A homology model of the venus flytrap domain of T1R3 shows the putative ligand binding site (yellow), predicted by alignment with ligand-contacting sites of rat mGluR1 (20), and mutations that confer sugar binding, which cluster in three distinct locations (red, green, and blue). 


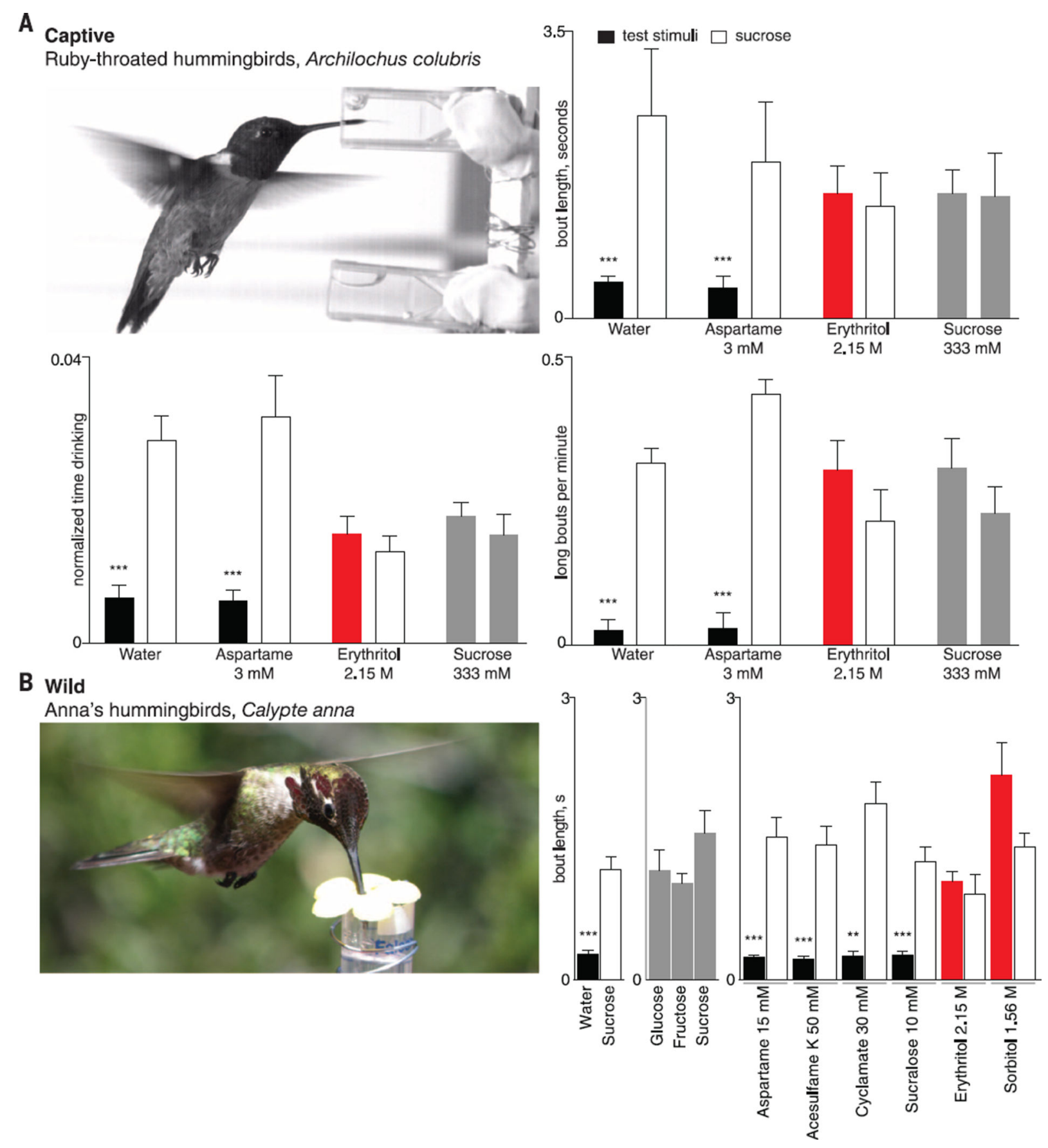

Fig. 4. T1R1-T1R3 agonists evoke taste responses in captive and wild hummingbirds

(A) Captive ruby-throated hummingbirds ( $n=3$ or 4 , mean \pm SE) were presented with solutions of test stimuli and sucrose $(333 \mathrm{mM})$, and the drinking bout lengths, time spent drinking, and number of long bouts ( $>1 \mathrm{~s}$ ) were recorded (linear mixed-effect models for differences between stimuli and sucrose, $* * * P \leq 0.001)$. Red bars indicate palatability similar to that of carbohydrates. (B) The taste preferences of wild Anna's hummingbirds were measured (mean bout lengths \pm SE, sample sizes: table S4, Kolmogorov-Smirnov tests for differences between stimuli and sucrose: $* P \leq 0.05$, **P $\leq 0.01$, ***P $\leq 0.001$ ). 
Concentrations: white, $500 \mathrm{mM}$; gray, $1 \mathrm{M}$; black, indicated. Red bars indicate equal preference. [Photo credits: (A) M.W.B. and F. Peaudecerf, (B) M.W.B.] 